\title{
Sitolojisi HGSIL Gelen Olgularda Kolposkopik Biopsi ile Eksizyonel İşlem Sonuçlarının Korelasyonu: 10 Yıllık Tersiyer Merkez Deneyimi
}

\author{
Correlation Between Colposcopic Biopsy and Excisional Procedure Results in Patients \\ with HGSIL Cytology: 10-Years Experience of A Tertiary Referral Centre
}

\section{Baki ERDEM, Uzm. Dr. " , Osman AŞICIOĞLU, Uzm. Dr. ", Gökçe TURAN, Uzm. Dr. ${ }^{2}$ İlkbal Temel YÜKSEL, Uzm. Dr ${ }^{1}$, Osman Samet GÜNKAYA, Uzm. Dr. ${ }^{2}$, İpek Yıldız ÖZAYDIN, Uzm. Dr. ${ }^{3}$, Işıl Şafak YILDIRIM ${ }^{4}$, Doğukan YILDIRIM ${ }^{1}$, Özgür AKBAYIR ${ }^{1}$}

1. Kanuni Sultan Süleyman Eğitim ve Araştırma Hastanesi, Jinekolojik Onkoloji Kliniği, İstanbul, Türkiye

2. Kanuni Sultan Süleyman Ĕgitim ve Araştırma Hastanesi, Kadın Hastalıkları ve Doğum Kliniği, İstanbul, Türkiye

3. Kanuni Sultan Süleyman Ĕgitim ve Araştırma Hastanesi, Patoloji Kliniği, İstanbul, Türkiye

4. Bağcllar Ĕ̆itim ve Araştırma Hastanesi, Kadın Hastalıkları ve Doğum Kliniği, İstanbul, Türkiye

\section{$\ddot{O Z Z E T}$}

Amaç: Sitoloji sonucu yüksek dereceli servikal intraepitelyal lezyon (HGSIL) olan olgularda, kolposkopik biopsi ve ekzisyonel ișlemler ile elde edilen patoloji sonuçları arasindaki korelasyonu incelemek.

Gereçler ve Yöntem: Toplamda 282 hastanın verilerine ulaşıl$d l$. Tüm hastalara kolposkopik muayene yapıldl. Kolposkopik biopsi sonucu invaziv kanser tanisl konulan 23 hasta klinik olarak evrelendi ve uygun tedavi planlandr (radikal histerektomi veya kemoradyoterapi). Diğer hastaların tümüne $(n=259$ eksizyonel işlemler (LEEP veya konizasyon) uygulandi. Her iki işlemden elde edilen patoloji sonuçlarindan en yüksek dereceli olan final patoloji olarak kaydedildi.

Bulgular: Kolposkopik biopsi ile eksizyonel islemler arasinda $\% 73,7$ oraninda $(n=191 / 259)$ uyum izlendi. 64 hastada $\leq$ CIN1 $(\% 22,7)$ bulunduğu ve bu grupta așırı tedavi yapıldığ tespit edildi. Hastalarin \%63, $5^{\prime} i(n=179) C I N 2+, \% 13,8^{\prime} i(n=39)$ ise invaziv kanser idi. Kolposkopik biopsi invaziv kanserli hastaların \%4l'inde (n=16/39) eksik patolojik taniya neden oldu. Kolposkopik biopsi ile eksizyonel ișlemler sonucu elde edilen patoloji sonuçlarındaki uyumu saptamak için kappa analizi yapıldı. $\kappa:$ 0,542 olarak hesapland ve bu değer ılıml derecede korelasyon anlamina gelmekteydi.

Sonuç: HGSIL sitoloji sonucu ile basvuran hastalarda kolposkopik biopsi ile eksizyonel işlemler arasinda yaklaşık 4 te 3 oraninda uyum bulunduğu tespit edilmiştir. CIN2+ ve invaziv kanser insidansının bu hastalarda yüksek bulunması nedeniyle,bu hastalarda biopsi sonucu $\leq$ CIN1 gelse bile eksizyonel işlem seçeneği sunulmasının daha doğru ve güvenli olacağını düşünmekteyiz.

Anahtar Kelimeler: hgsil, kolposkopi, servikal kanser

\section{ABSTRACT}

Objective: To evaluate the correlation between pathology results obtained by colposcopic biopsy and excisional procedures in cases with a cytology of high grade cervical intraepithelial lesion (HGSIL).

\section{İletișim}

Sorumlu Yazar: Baki ERDEM, Uzman Dr.

Adres: Kanuni Sultan Süleyman Eğitim ve Araştırma Hastanesi, Jinekolojik Onkoloji Kliniği, 34000, İstanbul, Türkiye

Tel: +90 (505) $4511893 \quad$ Fax: +90 (212) 5714790

E-Posta: drberdem@yandex.com

Makale Geliș: 14.09.2018

Makale Kabul: 21.03.2019

DOI: http://dx.doi.org/10.16948/zktipb.460069
Material and Methods: A total of 282 patients' data were collected. All patients underwent colposcopic examination. Twenty-three patients with a diagnosis of colposcopic biopsy-proven invasive cancer were clinically staged, and appropriate treatment was planned (radical hysterectomy or chemoradiotherapy). Excisional procedures were executed in all other patients $(n=259)$. The highest grade pathology obtained by biopsy or excisional procedures was recorded as the definitive final pathology.

Results: The agreement rate was $73.7 \%(n=191 / 259)$ between the colposcopic biopsy and excisional procedures. 64 patients had $\leq$ CIN1 (22.7\%), and overtreatment was found in this group. $63.5 \%$ of the patients $(n=179)$ were CIN $2+$, and $13.8 \%(n=$ 39) were invasive cancer. Colposcopic biopsy ended up with an incorrect pathologic diagnosis in $41 \%(n=16 / 39)$ of patients with invasive cancer. The kappa analysis was performed to determine the correlation between pathology results obtained by colposcopic biopsy and excisional procedures. $\kappa$ is calculated to be 0.542 , which means that the correlation was moderate.

Conclusion: It was determined that there were approximately three-quarters of agreement between colposcopic biopsy and excisional procedure results in patients with HGSIL cytology. Because of the high incidence of CIN2 + and invasive cancer in this group, we believe that it would be reasonable and safe to choose the excisional procedure option even if the biopsy result remains $\leq$ CIN1.

Keywords: cervical cancer, colposcopy, hgsil

\section{GíRiş}

Serviks kanseri dünyada kadınlarda en s1k görülen 4. kanser tipidir ve her yıl yaklaşık yarım milyon olguya tanı konulur (1). Gelișmemiș ülkelerde en s1k görülen jinekolojik kanser tipi serviks kanseridir ve olguların yaklaşık \%80'i bu ülkelerde görülmektedir (2). Gelişmiş ülkelerde ise tarama programları sayesinde serviks kanserinin insidansı ve mortalitesinde istikrarlı bir düşüş sağlanmıştır (3). Tarama programlarında en sık kullanılan yöntem Papanikolau (Pap) testidir. Pap smear'lerin \%90'1ndan fazlası "intraepitelyal lezyon veya malignite açısından negatif" olarak raporlanır (4). Pap smear testinin servikal preinvaziv lezyonları saptamada sensitivitesi $\% 79-100$, spesifisitesi ise $\% 30$ 80 arasında değismektedir $(5,6)$.

Pap testi yüksek dereceli skuamöz intraepitelyal lezyon (HGSIL) olarak yorumlanan hastalarda klasik yaklaşım kolposkopi eşliğinde yapılan punch 
biopsidir. HGSIL sitolojinin, teoride histopatolojik olarak servikal intraepitelyal neoplazi (CIN) 2 veya 3 ile uyum göstereceği varsayılmaktadır. Fakat kolposkopik biopsi ile alınan doku parçası genellikle $5 \mathrm{~mm}$ 'den küçüktür ve her zaman en şiddetli servikal lezyondan biopsi yapılamayabilir. Bir çalışmada HGSIL smearlerin sadece \%43-66'sında biopsi ile CIN2 veya CIN3 tanıs1 doğrulanabilmiştir (7). Bu nedenle bu hastaların çoğuna nihayetinde eksizyonel işlemler uygulanmaktadır (8).

$\mathrm{Bu}$ çalışmadaki amacımız kliniğimizde başvuran ve sitoloji sonucu HGSIL olan kadinlarda, kolposkopik biopsi bulguları ile ekzisyonel işlemler sonucu elde edilen patoloji bulguları arasındaki korelasyonu incelemekti.

\section{GEREÇLER ve YÖNTEM}

Çalışma Kanuni Eğitim ve Araștırma Hastanesi'nde gerçekleştirildi. Kliniğin elektronik veri tabanı (Filemaker Pro 6, Filemaker, Inc) retrospektif olaraktaranarak, 2008-2018 y1lları arasında HGSIL sitoloji sonucu ile başvuran hastaların bilgilerine ulaşıldı. Bilgilerinin tamamına ulaşılamayan ve gebe olan hastalar çalışma dışı bırakı1dı. 282 hasta çalışmaya dahil edildi. Bu hastaların tümüne kolposkopik muayene yapıldı; şüpheli alanlardan biopsi alındı ve endoservikal küretaj (ECC) uyguland1. Kolposkopik muayenede şüpheli lezyon izlenmeyen hastalarda ise sadece ECC uygulandi. Biopsi sonucunda elde edilen patoloji bulgular1; $\leq \mathrm{CIN} 1, \mathrm{CIN} 2+$ (CIN2, CIN3, insitu kanser) veya invaziv kanser olarak kaydedildi. Kolposkopik biopsi sonucu invaziv kanser tanısı konulan 23 hasta klinik olarak evrelendi ve uygun tedavi planland1 (radikal histerektomi veyakemoradyoterapi). Diğer hastaların tümüne $(\mathrm{n}=259)$ eksizyonel işlemler (loop elektrocerrahi eksizyon prosedürü (LEEP) veya soğuk konizasyon) uyguland1. Eksizyonel işlem sonucu elde edilen patoloji bulgular1; $\leq \mathrm{CIN} 1, \mathrm{CIN} 2+$ veya invaziv kanser olarak kaydedildi. Kolposkopik biopsi veya eksizyonel işlemlerden elde edilen patoloji sonuçlarından en yüksek grade'li olanı final patoloji olarak kaydedildi.

\section{Istatistiksel analiz}

İstatistiksel işlemlerin analizi için SPSS 22.0.0.0 kullanild1.

\section{BULGULAR}

Hastaların yas ortalamas $141,9 \pm 9,6$, ortalama paritesi $2,6 \pm 1,7$ idi. Hastaların $\% 78,4$ 'ü $(n=221)$ premenopozaldi. 282 hastaya kolposkopik biopsi yapılırken, 259 hastaya eksizyonel işlemler uygulandı. Eksizyonel işlemlerin 102'si $(\% 39,4)$ konizasyon, 157'si $(\% 60,6)$ ise LEEP idi. Kolposkopik biopsi ile eksizyonel işlemler arasında tüm grupta $\% 73,7$ oranında $(\mathrm{n}=191 / 259)$ uyum izlendi. $\mathrm{Bu}$ oran $\leq \mathrm{CIN} 1$ grubunda \%66 iken, CIN2+ grubunda $\% 78,4$ idi. (Tablo 1). 47 hastada $(\% 18,2)$ patolojik grade artış1 izlendi. 21 hastada $(\% 8,1)$ ise eksizyonel işlemler ile daha düşük grade elde edildi. Final patoloji sonuçları değerlendirildiğinde 64 hastada $\leq$ CIN1 $(\% 22,7)$ bulunduğu ve bu grupta aşırı tedavi yapıldığ 1 tespit edildi. Hastaların $\% 63,5$ 'i $(n=179)$ CIN2+, \%13,8'i (n=39) ise invaziv kanser idi (Tablo 2). Kolposkopik biopsi invaziv kanserli hastaların \%41'inde $(n=16 / 39)$ eksik patolojik taniya neden oldu; 14 hastada CIN2+, 2 hastada ise $\leq$ CIN1 lezyon saptayabilmişti. Kolposkopik biopsi ile eksizyonel işlemler sonucu elde edilen patoloji sonuçlarındaki uyumu saptamak için kappa analizi yapıld1. $\kappa: ~ 0,542$ olarak hesapland 1 ve bu değer 1lım11 derecede korelasyon anlamına gelmekteydi.

İnvaziv kanser saptanan 39 hastanın 14 tanesi evre $1 \mathrm{~B} 2$ veya $2 \mathrm{~B}$ olarak değerlendirildi ve kemoradyoterapiye yönlendirildi. 15 hastaya radikal histerektomi yapıld1. 10 hastaya basit histerektomi yapild.

Tablo 1: Kolposkopikbiopsi ile eksizyonel işlem sonuçlarının karşılaştırilmasi.

\begin{tabular}{|c|c|c|c|c|c|}
\hline \multicolumn{7}{|c|}{ Konizasyon / LEEP } \\
\hline Biopsi \pm ECC & $\begin{array}{c}\leq \text { CIN 1 } \\
(\mathrm{n})\end{array}$ & $\begin{array}{c}\text { CIN 2+i } \\
(\mathrm{n})\end{array}$ & $\begin{array}{c}\text { İnvaziv } \\
\text { kanser (n) }\end{array}$ & $\begin{array}{c}\text { Toplam } \\
(\mathrm{n})\end{array}$ & $\begin{array}{c}\text { Uyum } \\
\text { oran }\end{array}$ \\
\hline$\leq$ CIN 1 (n) & 64 & 31 & 2 & 97 & $\% 66$ \\
\hline CIN 2+ (n) & 21 & 127 & 14 & 162 & $\% 78,4$ \\
\hline Toplam (n) & 85 & 158 & 16 & 259 & $\% 73,7$ \\
\hline
\end{tabular}

Tablo 2: Final patoloji bulguları.

\begin{tabular}{|c|c|c|c|}
\hline$\leq \mathrm{CIN} 1(\mathrm{n})$ & $\mathrm{CIN} 2+(\mathrm{n})$ & İnvaziv kanser $(\mathrm{n})$ & Toplam \\
\hline $64(\% 22,7)$ & $179(\% 63,5)$ & $39(\% 13,8)$ & 282 \\
\hline
\end{tabular}

\section{TARTIȘMA}

Servikal intraepitelyal neoplaziler(CIN), derecesine bağlı olarak invaziv kansere ilerleyebilirler (9). Sitolojik tarama ile bu preinvaziv lezyonların erken tanıs1 ve tedavisi mümkün olmuş ve serviks kanserinin insidansında düşme sağlanmıştır (10). HGSIL sitolojisi olan hastaların yaklaşık üçte ikisinde CIN 2 veya CIN 3 lezyon, \%2'sinde ise invaziv kanser bulunmaktadır (7). Bu riskli grupta yapilan kolposkopik biopsi sonucu CIN 2 veya CIN 3 gelmesi durumunda siklıkla LEEP veya konizasyon gibi eksizyonel işlemler önerilir (11). HGSIL sitolojisi olan ve kolposkopik biopsi sonucu $\leq \mathrm{CIN} 1$ gelen hastalar ise klinisyenleri ikilemde birakmaktadir. $\mathrm{Bu}$ grupta Amerikan Kolposkopi ve Servikal Patoloji Cemiyeti (ASCCP) geçmiş yıllarda tanı ve tedavi amacıyla eksizyonel işlemlerin kullanılmasını önermekteydi (12). Fakat en güncel ASCCP k1lavuzunda bu grup hastalarda tanısal eksizyonel işlemlerin yanı sira, 12 ve 24 . aylarda kotest yapılması veya sitoloji, histoloji ve kolposkopi bulgularının tekrar gözden geçirilmesinin de önerilebileceği bildirilmiştir (11). CIN tanısında kolposkopik bulgular ve biopsi ile elde edilen histopatoloji sonuçları, bahsedilen k1lavuzlarda önerilen yönetim algoritmalarının şekillenmesini sağlamaktadır.

Literatürde kolposkopik biopsi ile eksizyon spesimeni arasında \%43,3-85,8 arasında değişen bir uyum oranı bildirilmektedir. $(5,13-18)$. Güncel retrospektif kohort çalışmamızdatüm grupta \% 73,7 $(n=191 / 259)$ oranında uyum saptadık. Kolposkopik biyopsinin yüksek dereceli lezyonlarda düşük dereceli lezyonlara göre daha uyumlu sonuçlar verdiği bildirilmiştir (5). Bizim çalışmamızda da uyum ora- 
n1 benzer şekilde CIN2+ grubunda $\leq \mathrm{CIN} 1$ grubuna göre daha yüksek bulunmuştur (\%78,4 vs. \%66). Çalışmamızda HGSIL sitolojisi olan kadınların çoğunluğunda $(\% 63,5)$ final patolojide CIN2+ lezyon saptand1. $\mathrm{Bu}$ bulgumuz da literatür ile uyumludur (7).

Howe ve Vincenti, LEEP ile elde edilen patoloji sonuçlarının kolposkopik biopsiye göre \%24 oranında daha ileri olduğunu ve LEEP işleminin CIN tanısını arttırdığını bildirmişlerdir (15).

Byroom ve arkadaşları benzer şekilde bu oranı $\% 20,5$ olarak tespit etmiştir (19). Jung ve arkadaşları, yüksek vaka sayısına sahip güncel çalışmalarında bu oran $\% 23,1$ olarak bildirmişlerdir (18). Bizim çalışmamızda ise hastalarımızın \% 18,2'sinde $(n=47 / 259)$ eksizyonel işlemler ile daha ileri grade elde edilmiştir.Kolposkopik biopsi ile CIN2+ tanisı konulan 162 hastanın 14'ünde $(\% 8,6)$ invaziv kanser vardı. Jung ve arkadașları bu grupta invaziv kanser oranını \% 15,2 olarak tespit etmiștir (18). Biopsi ile eksizyonel işlemler arasındaki uyumsuzlugun nedenleri arasında; kolposkopistin deneyimsiz olması, doğru yerden biopsi alınamaması ve lezyonun her olguda kolposkopi ile görünür olmaması bulunmaktadır (20). Çalışmamızda kolposkopik biopsinin $\leq$ CIN1 olarak tanı koyduğu 97 hastanın 31 'inde $(\% 31,9)$ eksizyonel işlemler ile CIN2+, 2 'sinde $(\% 2,1)$ ise invaziv kanser saptand. Kolposkopik biopsi ile $\leq$ CIN1 olarak eksik tanı konulan bu hasta grubu özellikle endișe vericidir, çünkü güncel kılavuzlarda bu grup hastalar için gözlemsel stratejilerin kullanımı seçenek olarak sunulmaktadır (11).

Çalışmamızda hastaların \%8,1'inde $(\mathrm{n}=21)$ kolposkopik biopside CIN2+ hastalık varken eksizyonel ișlemlerde $\leq \mathrm{CIN} 1$ lezyon bulunmuștur. $\mathrm{Bu}$ oran literatürde $\% 7-33,6$ arasında bildirilmiştir $(17,18,21,22)$. Lezyonun küçük olması ve biopsi ile total olarak çıartılması, biopsiye sekonder gelișen inflamatuvar reaksiyonun lezyonda regresyona yol açması ve eksizyonel işlem ile lezyonun çıkartılamaması bu durumun sebepleri arasındadır $(5,17,22)$. Çalıșmamızda hastaların \%22,7'sinde $(\mathrm{n}=64 / 282)$ hem kolposkopik biopsi sonucu,hem de eksizyonel işlem sonucu $\leq$ CIN1 olarak saptandı ve bu grupta aşırı tedavi uygulanmıştı. Aşırı tedavi oran1 literatürde \%11-35 arasında bildirilmektedir (23). HGSIL Pap smearlerde bildirilen \%8-12 arasındaki yanlış pozitifliğin final patolojide $\leq$ CIN1 lezyonu olan hastaların sayısına katkıda bulunacağı unutulmamalidir (24).

Kliniğimizin servikal neoplaziler için refere bir merkez olması nedeniyle, HGSIL sitoloji sonuçlarının bir kısmının dış merkezlerden elde edilmiş olması calıșmamızın limitasyonlarından birisidir. Bilindiği gibi patologların Pap smear'leri değerlendirmesinde farklılıklar bulunmaktadır (25). Biopsi ve eksizyonel işlemler sonucu elde edilen sonuçlar hastanemizde birden fazla patolog tarafindan değerlendirilmiştir ve bu durum jinekopatologlar arasinda bile farklı sonuçlara yol açabilmektedir (26). Çalışmamızın diğer bir limitasyonu kolposkopik biopsilerin birden fazla uygulayıcı tarafindan yapılmış olması ve bu uygulayıcıların önemli bir kısmının jinekolojik onkoloji yan dal eğitim sürecinde olan uzmanlardan oluşmasıdır.

\section{SONUC}

Biz bu çalıșmamızda, özellikle CIN açısından yüksek riskli olan bir hasta kohortunu; HGSIL sitolojisi bulunan kadınların kolposkopik biopsi ve eksizyonel işlem ile elde edilen histoloji sonuçlarını karşılaştırdık. Bulgularımız sitoloji sonucu HGSIL olan hastalarda kolposkopik biopsinin CIN2+ ve hatta invaziv kanserleri bile atlayabildiğini göstermiştir. Bu hastalarda sadece kolposkopik biopsi sonucuna güvenip ASCCP kılavuzunun 12. ay kotest seçeneğini önermiş olsaydık, gerçek CIN2+ hastaların yaklaşık beşte birini $(\mathrm{n}=31 / 158, \% 19,6)$, invaziv kanserlerin ise $\% 5$ 'ini $(n=2 / 39)$ atlamıș olacaktık. Bu nedenle HGSIL sitoloji sonucu olan hastalara biopsi sonucu $\leq$ CIN1 gelse bile eksizyonel işlem seçeneği sunulmasının daha doğru ve güvenli olacağını düşünmekteyiz.

\section{KAYNAKLAR}

1. 1-Ferlay J, Soerjomataram I, Ervik M, et al. GLOBOCAN 2012 V1.0, Cancer Incidence and Mortality Worldwide: IARC CancerBase No. 11. (http://globocan.iarc.fr). Accessed 16 July 2014.

2-Guidozzi F. Screening for cervical cancer. Obstet Gynecol Surv 1996;51(4):247-52.

3-Waxman A.G., Zsemkye M.M.: "Preventing cervical cancer: the Pap test and the HPV vacCINe". Med. Clin. North. Am., 2008, 92, 1059.

4-Joste N.: "Overview of the cytology laboratory: specimen processing through diagnosis". Obstet. Gynecol. Clin. North Am., 2008, 35, 549 .

5-Duesing N, Schwarz J, Choschzick M, et al. Assessment of cervical intraepithelial neoplasia (CIN) with colposcopic biopsy and efficacy of loop electrosurgical excision procedure (LEEP). Arch Gynecol Obstet 2012;286:1549-54

6-Labani S, Asthana S. Age-specific performance of careHPV versus Papanicolaou and visual inspection of cervix with acetic acid testing in a primary cervical cancer screening. J Epidemiol Community Health 2015; 2015:205851.

7-L. S. Massad, Y. C. Collins, and P. M. Meyer, "Biopsy correlates of abnormal cervical cytology classified using the bethesda system, " Gynecologic Oncology, vol. 82, no. 3, pp. 516-522, 2001.

8-Wright T.C. Jr., Massad L.S., Dunton C.J., Spitzer M., Wilkinson E.J., Solomon D.; 2006 American Society for Colposcopy and Cervical Pathology-sponsored Consensus Conference: "2006 consensus guidelines for the management of women with abnormal cervical cancer screening tests". Am. J. Obstet. Gynecol., 2007, 197, 346.

9-Ostor AG. Natural history of cervical intraepithelial neoplasia: a critical review. Int J Gynecol Pathol 1993;12:186-92.

10-Sasieni P, Adams J. Effect of screening on cervical cancer mortality in England and Wales: analysis of trends with an age period cohort model. BMJ 1999;318:1244-5.

11- Massad LS, Einstein MH, Huh WK, Katki HA, Kinney WK, Schiffman M, et al. 2012 updated consensus guidelines for the management of abnormal cervical cancer screening tests and cancer precursors. Obstet Gynecol 2013;121:829-46. 
12- T. C. Wright Jr., J. T. Cox, L. S. Massad, L. B. Twiggs, and E. J. Wilkinson, "2001 consensus guidelines for the management of women with cervical cytological abnormalities," Journal of Lower Genital Tract Disease, vol. 6, no. 2, pp. 127-143, 2002

13- Buxton EJ, Luesley DM, Shafi MI et al. Colposcopically directed punch biopsy: a potentially misleading investigation. Br J Obstet Gynaecol 1991;98:1273-6.

14- Shehan M, Soutter W, Lim K et al. Reliability of colposcopy and directed punch biopsy. Br J Obstet Gynaecol 1990;97:8116

15- Howe D, Vincenti A. Is large loop excision of the transformation zone (LLETZ) more accurate than colposcopically directed punch biopsy in the diagnosis of cervical intraepithelial neoplasia? Br J Obstet Gynaecol 1991;98:588-91.

16- Bonardi R, Cecchini S, Grazzini G et al. Loop electrosurgical excision procedure of the transformation zone and colposcopically directed punch biopsy in the diagnosis of cervical lesions. Obstet Gynecol 1992;80:1020-2.

17- Barker B, Garcia F, Lozevski J, Warner J, Hatch K. The correlation between colposcopically directed cervical biopsy and loop electrosurgical excision procedure pathology and the effect of time on that agreement. Gynecol Oncol 2001; 82: $22-6$.

18-Jung Y, Lee AR, Lee SJ, Lee YS, Park DC, Park EK. Clinical factors that affect diagnostic discrepancy between colposcopically directed biopsies and loop electrosurgical excision procedure conization of the uterine cervix. Obstet Gynecol Sci 2018;61(4):477-488
19- Byrom J, Douce G, Jones PW, et al. Should punch biopsies be used when high-grade disease is suspected at initial colposcopic assessment? a prospective study. Int J Gynecol Cancer 2006;16(1):253-6.

20- Underwood M, Arbyn M, Parry-Smith W, et al. Accuracy of colposcopy-directed punch biopsies: a systematic review and meta-analysis. BJOG 2012;119:1293-301.

21- Berdishevsky L, Karmin R, Chuang L. Treatment of highgrade squamous intraepithelial lesions: a 2-versus 3-step approach. Am J Obstet Gynecol. 2004;/190:/1424_6.

22- Ryu A, Nam K, Chung S, et al. Absence of dysplasia in the excised cervix by a loop electrosurgical excision procedure in the treatment of cervical intraepithelial neoplasia. J Gynecol Oncol 2010;21:87-92.

23- Ebisch RMF, Rovers MM, Bosgraaf RP, van der Pluijm-Schouten HW, Melchers WJG, van den Akker PAJ, et al. Evidence supporting see-and-treat management of cervical intraepithelial neoplasia: a systematic review and meta-analysis. BJOG. 2016; 123(1): 59-66.

24-Mount S, Harmon M, Eltabbakh G, Uyar D, Leiman G. False positive diagnosis in conventional and liquid-based cytology specimens. Acta Cytol 2004;48:363Y71.

25- M. H. Stoler and M. Schiffman, "Interobserver reproducibility of cervical cytologic and histologic interpretations: realistic estimates from the ASCUS-LSIL Triage Study," Journal of the American Medical Association, vol. 285, no. 11, pp. 1500-1505, 2001. 26-McCluggage WE, Bharucha H, Caughley LM, Date A, Hamilton PW, Thornton CM, Walsh MY. Interobserver variation in the reporting of cervical colposcopic biopsy specimens: comparison of grading systems. J Clin Pathol 1996;49:833-5. 